\title{
AUTOMATIC MATCHING OF LARGE SCALE IMAGES AND TERRESTRIAL LIDAR BASED ON APP SYNERGY OF MOBILE PHONE
}

\author{
Guofang Xia ${ }^{1,2,3}$, Chunmei $\mathrm{Hu}^{1,2,3} *$ \\ ${ }^{1}$ School of Geomantic and Urban Information, Beijing University of Civil Engineering and Architecture, NO.15Yongyuan \\ Road, Daxing District, Beijing, 102616 - (Guofang Xia, Chunmei Hu)@ bucea.edu.cn \\ ${ }^{2}$ Beijing, Key Laboratory for fine reconstruction and health monitoring of Architectural Heritage, NO.15Yongyuan \\ Road, Daxing District, Beijing, 102616 \\ ${ }^{3}$ Engineering Research Center of Representative Building and Architectural Heritage Database, Ministry of Education, Beijing,
} 102616, China

Commission III, WG III/6

KEY WORDS: Mobile phone APP, Images, Laser Point Cloud, Matching

\begin{abstract}
:
The digitalization of Cultural Heritage based on ground laser scanning technology has been widely applied. High-precision scanning and high-resolution photography of cultural relics are the main methods of data acquisition. The reconstruction with the complete point cloud and high-resolution image requires the matching of image and point cloud, the acquisition of the homonym feature points, the data registration, etc. However, the one-to-one correspondence between image and corresponding point cloud depends on inefficient manual search. The effective classify and management of a large number of image and the matching of large image and corresponding point cloud will be the focus of the research. In this paper, we propose automatic matching of large scale images and terrestrial LiDAR based on APP synergy of mobile phone. Firstly, we develop an APP based on Android, take pictures and record related information of classification. Secondly, all the images are automatically grouped with the recorded information. Thirdly, the matching algorithm is used to match the global and local image. According to the one-to-one correspondence between the global image and the point cloud reflection intensity image, the automatic matching of the image and its corresponding laser radar point cloud is realized. Finally, the mapping relationship between global image, local image and intensity image is established according to homonym feature point. So we can establish the data structure of the global image, the local image in the global image, the local image corresponding point cloud, and carry on the visualization management and query of image.
\end{abstract}

\section{INTRODUCTION}

High-resolution image and laser scanning data are the main data sources of digitalization of cultural heritage at present. Large objects like ancient buildings, have a large number of highresolution images, therefore, the main contents of the project are the grouping, management, and the one-to-one correspondence with the corresponding point cloud of the acquired images.

Because of manual completion currently in this part, the way of image management and query still needs a certain time to consume. From image acquisition, image grouping, image management query, image and point cloud, the efficiency of this part will be greatly improved if the image automation grouping, the large-scale image visualization query, the image and the point cloud matching can be realized. With the development of smartphone, it provides more access to information. More and more functions of APP are developed and applied on mobile phone. In this paper, the data is obtained by the mobile phone APP cooperative photographing system, and the large scale high-resolution point cloud is automatically grouped. For each group of data, the location relationship between the whole image of the object and the local image of it is obtained by image matching, and the location of the local image in the global image is obtained by affine transformation, and the image and the corresponding point cloud are automatically matched through the global image and its corresponding point cloud reflection intensity image. In image management, the topological relationship between global image and local influence is stored to realize visual management and query of large-scale images.

\section{METHOD}

\subsection{The image grading block acquisition of object}

In order to perform subsequent block visualization management and query on the image, it is necessary to design the classification of image acquisition before obtaining image of large-scale objects. Firstly, we design the shooting sequence for multi-body objects and number them. Secondly, according to the characteristics of the single object, it is divided into different blocks for photography. For large buildings, blocks can be divided according to the internal and external methods of south, east, north and west, moreover, the blocks are named according to the name and orientation of the object. The ground object usually chooses the digital camera to obtain image. According to the surrounding environment of the object, we select the appropriate focal length lens, and determine the photography distance on the basis of the required image resolution and the overlap degree on the basis of the single image and the overlapping image photography. Besides, we should realize the visualization management of the object image

\footnotetext{
* Corresponding author. E-mail addresses: huchunmei@ bucea.edu.cn (Chunmei Hu)
} 
classification. When we are shooting high resolution images, we take the global image of the block according to the classified object in order to establish the topological relationship between the whole and the local image of the block region in the followup.

\subsection{Mobile phone APP cooperative photographing system}

Currently, many mobile phone operating systems have been introduced by many domestic and foreign manufacturers. The mainstream mobile operating systems are roughly Symbian OS, iOS, Android, Windows Mobile, PalmOS, and Blackberry, etc. The Android platform has development capabilities. Its product interface is user-friendly, and it has touch screens, advanced graphic displays and other functions. It allows each vendor to develop and introduce their own features as well as unique applications.

Android is a software platform and operating system based on the Linux kernel. It uses software stack architecture and Java programming language to write. The hierarchical architecture is divided into four levels from high to low. First is application layer. Second is application framework layer. Third is system runtime layer. Four is the core layer of Linux. Android features include application architecture, virtual machine Dalvik, browser integration, further optimization of drawing capabilities, database SQLite, multimedia support, global communication technology, Bluetooth, EDGE, 3G and Wi-Fi, global positioning systems. An Android application consists of four parts: Activity, Intent Receiver, Service, and Content Provider. Each type of four different application components serves different purposes, has different life cycles, and defines how to create and destroy. These four basic components run in the background of the system. Some of them are visible views, some are invisible views, and four basic components are four views. But not every Android application is made up of these four parts. When used in an application, it needs to be configured in the configuration file Android Mainfest.xml, which is required for every Android application and is used to define the application's components and its functions, as well as the necessary conditions, and so on.

2.2.1 Development function design: The main interface of the system mainly manages all functions which can be divided into seven functions. The Project Name module is used to enter the project name and local name to build the new project. The Global and Local module is used to record the ratios of the global photo and local photo. The Project Image module uses a mobile phone camera to take photos which are automatically saved to the folder. The Start module is used to record the time when we start taking pictures. The Full Picture Time module is used to record the time when the global picture was taken. The End module is used to record the time when we finish taking the pictures. The View module is used to view the contents of the configuration file which record time information and ratio information. Its function design is shown in the following Figure 1.

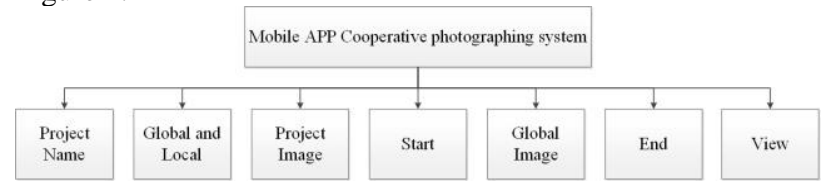

Figure 1. Function Design
2.2.2 Data Design: First is data content. The data content involved mainly includes image data obtained by photographing, and text data which record the time. There are two sources of image data. The Imagenote folder is used to save images shot by mobile phone app. The Photo folder is used to save the image taken by the SLR camera. The mobile phone APP generates text data which record the photographing time, the ratio value of the global and the local. The Imagenote folder is also used to save the document that records the project information. The document is divided into four lines, which are the time when we shoot the global image, the time when we start, the time when we finish, and the ratio value of the global and the local. Second is data format. Image data in JPG format and Document data in TXT format. The system interface is shown in the following Figure 2.

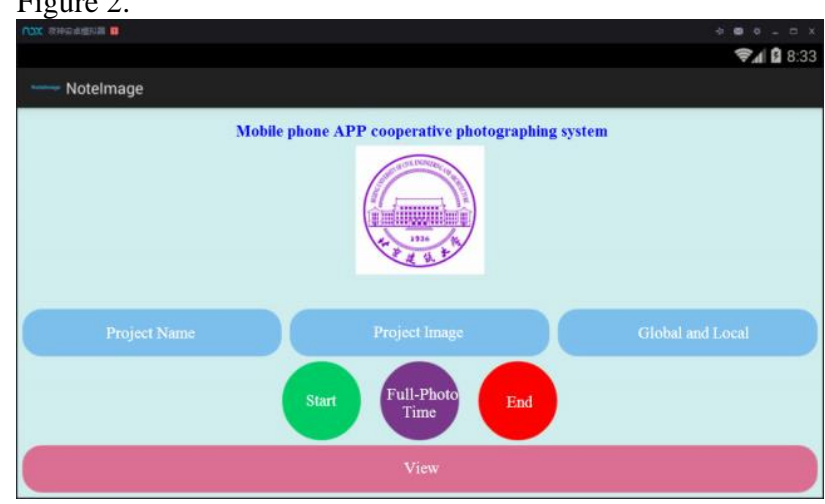

Figure 2. The system interface

The key technologies are three sizes and three variants in the development of the Android camera. The three dimensions are the size of the Surfaceview, the size of the Picturesize, and the size of the Previewsize. Surfaceview is used to preview the Camera, and the size of its full screen is the same size as the Screen. The size of Picturesize is the size after taking pictures. The size of the preview size is the size of the preview frame data. The three kinds of distortion is used to preview the length and width distortion of objects, the deformation of objects in the picture and the moment when we take picture, and preview that the picture will suddenly deform and revert to the normal. The latter two kinds of deformation are caused by the inconsistency between Previewsize and Picturesize's ratio of length to width. So we need to ensure that the length to width ratio of preview Surfaceview, Previewsize and Picturesize should be consistent.

In the process of image acquisition, we name the block according to the result of object grading and block, and build the APP engineering with the same name. Then we take photos according to the designed distance and overlap, and set the color card, adjust the sensitivity and exposure time according to the shooting environment at the moment. At the beginning of taking block photos, the start time is recorded. When shooting the block global image, we click the corresponding button to record the time, and input the ratio of the resolution difference of the global image to the local image. After the block is taken, the end button is clicked. At this time, APP records the corresponding photo information of a block. Although the information of this APP record is simple, it is of great help to the subsequent automatic grouping of photos. Furthermore, the carrier of APP is mobile phone which is convenient to carry and use. So it is very suitable for engineering practice. 


\subsection{Image Grouping based on APP Synergetic Recording Information}

The image automatic grouping system is suitable for reading the captured images and configuration files. They are automatically matched and assigned to corresponding folders. Its main functions are as shown in the following Figure 3.

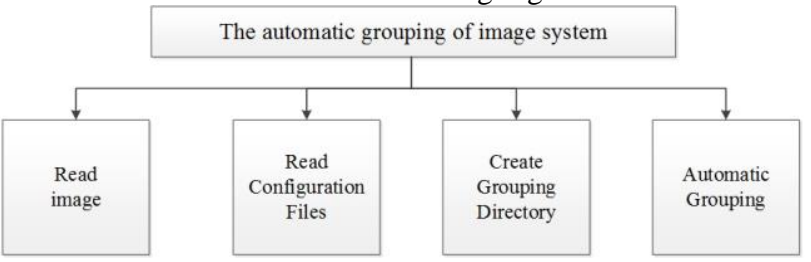

Figure 3. The main functions of the system

2.3.1 System Composition: The main function modules of image automatic grouping system are reading image module and reading group module. Among the reading group module, it provides the function of reading configuration files, creating grouping directory and automatic grouping (Figure 5).

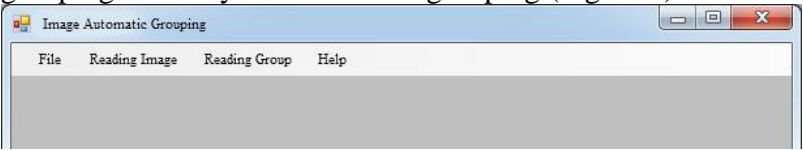

Figure 4. System master interface

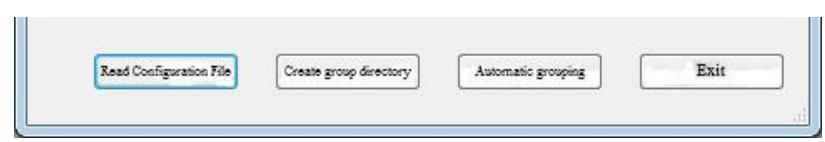

Figure 5. The Reading Group

Automatic grouping system automatically groups images according to the segmentation and naming of the photographed objects, instead of manual grouping, which greatly improves the efficiency of image sorting.

\subsection{Multi-image automatic location and matching with point cloud data}

We have automatically grouped images in mass images according to the blocks of the photographed objects by using APP recording information, but the query of the images and the manual operation to the corresponding point cloud data still affect efficiency of the engineering. To solve these problems, the location of local images in the whole image is determined according to the matching of the whole and local image data of the object. Then a one-to-one correspondence between local image and point cloud is established based on the reflected intensity image of object point cloud.

SIFT algorithm was put forward by Canadian professor David G.Lowe in 1999, and it is a partial description operator of image In 2004, it was once again completed by Professor David G.Lowe, and it was published in International Journal of Cumputer Vision (IJCV).

The characteristics of the algorithm are independent of the size and rotation of the image, and the tolerance to changes in light, noise, and micro-angle of view is also quite high. Therefore, using this algorithm in the field of image processing can effectively solve problems and obtain relatively good results. This paper applies SIFT matching algorithm to achieve the global image and local image matching, and applies RANSAC algorithm to eliminate the false matching. The affine transform function is set up by using the matching result, and the local image is mapped to the global image to establish its outer quadrilateral. At the same time, the topological relationship between the global image and the local image is established.

Before the photographing, the photographing object is divided into blocks. The entire point cloud data of the object is also segmented according to the content of the block. The reflection intensity image is generated according to the reflection intensity of the divided point cloud, and the image is in one-to-one correspondence with the global image of the area. Based on the above-mentioned topological relationship between the local image and the global image, a one-to-one correspondence between the local image and reflection intensity image of the point cloud is established. According to the image area on the reflection intensity image of the point cloud, we determine the corresponding $3 \mathrm{D}$ point cloud data and complete the matching between local image and point cloud data.

\subsection{Visual Query of Image}

The topological image data includes the name and path of the target image, and the location of the largest circumscribed rectangle of the target image boundary in the search area. The simplest topological image data format is to store the information contained in the data in the form of file streams. In the visual query of images, range search can be performed to stored documents by obtaining mouse position information. Another method is to establish a database specifically for the relationship between the search area image and the target area image. In the query function, the topological relationship of the image is stored in the database. Compared to simply using file streams for storage, the establishment of a database makes data storage more standardized and can greatly increase the speed of operation.

When the target image file is imported, the topological relationship between each target image and the search area image will be uniformly saved. At the same time, the boundary of the target image is also drawn on the search area image. When the user selects in the visual interface, the program will obtain the coordinate of position point which the user's mouse click, and screen in the stored topology relationship. When the point coordinates meet the boundary coordinates of a certain target image, the target image's attribute information will be obtained. The process is as shown in the Figure 6.

Obtain the coordinate of position point which the user' s mouse click

Comparison of coordinate with high-resolution coordinate

Extract the name, boundary and location of the high-resolution image in the boundary coordinate, and display it

Figure 6. The Flow chart 


\section{EXPERIMENTS AND ANALYSIS}

3.1 Mobile phone APP collaborative photographing and image grouping

(1) Open the Mobile phone APP and enter the main interface (Figure 7).

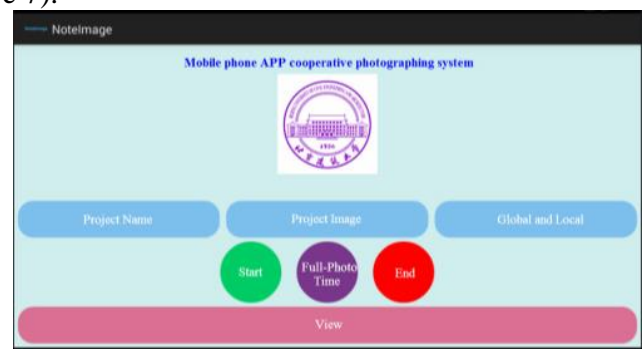

Figure 7. The main interface of Mobile APP

(2) Click the "Project Name" and enter: Project name and local name (Figure 8).

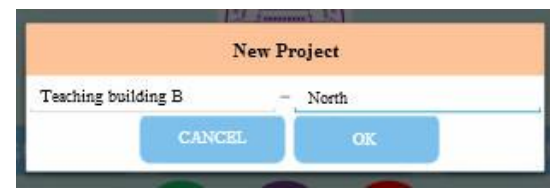

Figure 8. New project

(3) Click the "OK" button to create a new success.

(4) Click the "Start" button to start taking pictures with a SLR camera.

(5) When shooting a global view of a building, click the "Global Image" button to record the time.

(6) When shooting is complete, we click the "Finish" button. Click the "global / local" button and enter the scale factor (Figure 9).

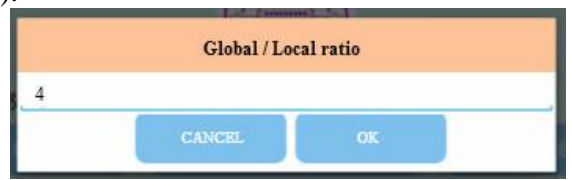

Figure 9. Global / local ratio

(7) Follow this method to take all the photos. After the shooting, click the "View" button to see the records of all the project (Figure 10).

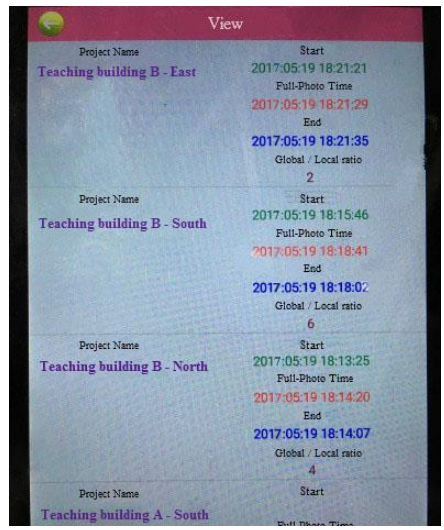

Figure 10. Check all the projects

(8) Import captured images (.jpg) into a folder.

(9) Export documents (.txt) that record project information from the phone (Figure 11).

$$
\begin{aligned}
& \text { Teaching building B - East } \\
& \text { Teaching building B - North } \\
& \text { Teaching building B - South }
\end{aligned}
$$

Figure 11. The configuration file

(10) Open automatic grouping system of the image and enter the main interface.

(11) Click "Open Image".

(12) Load the shot image.

(13) View the shooting image and time (Figure 12).

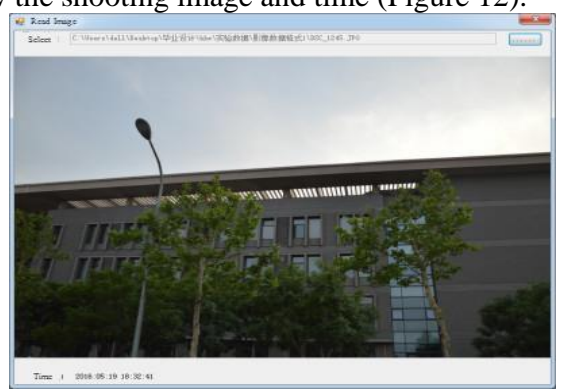

Figure 12. View Image

(14) Click "Read group information".

(15) Click "Read Configuration File" to read the file that records the configuration information (Figure 13).

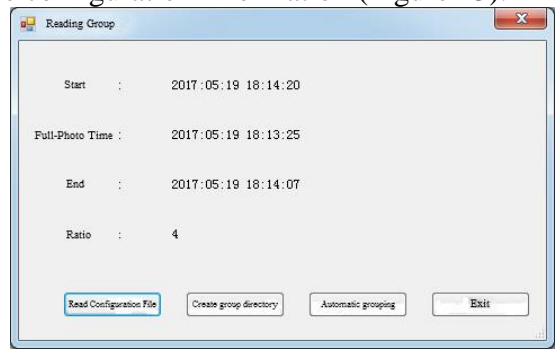

Figure 13. View the configuration file

(16) Click "Create group directory", the system automatically generates a folder with the same text name, at the same time under this folder create "global" folder and "local" folder.

(17) Click "Automatic grouping", the system will automatically match the information and classify the captured image (Figure 14).

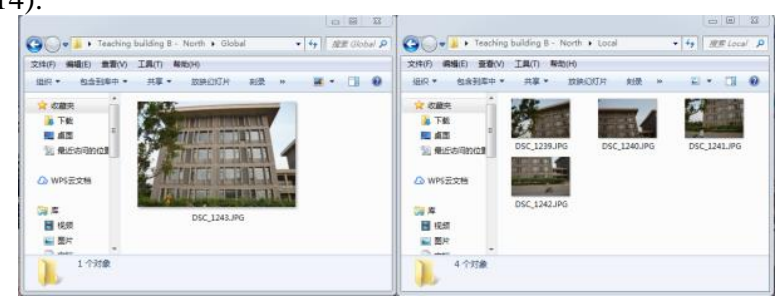

Figure 14. Automatic grouping 


\subsection{One-to-one correspondence between images and point} clouds and visual management

The function modules of Multi-image positioning grouping and visual query system are shown in the Figure 15.

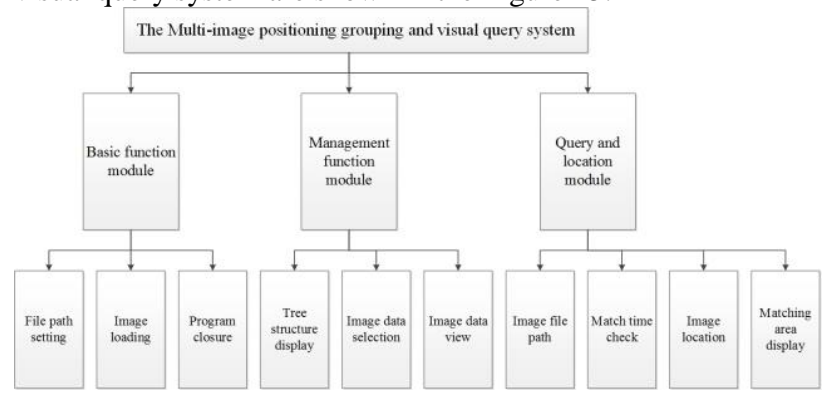

Figure 15 . The functional modules of the system

The matching result between the local image and the global image of an object is as follows (Figure 16).

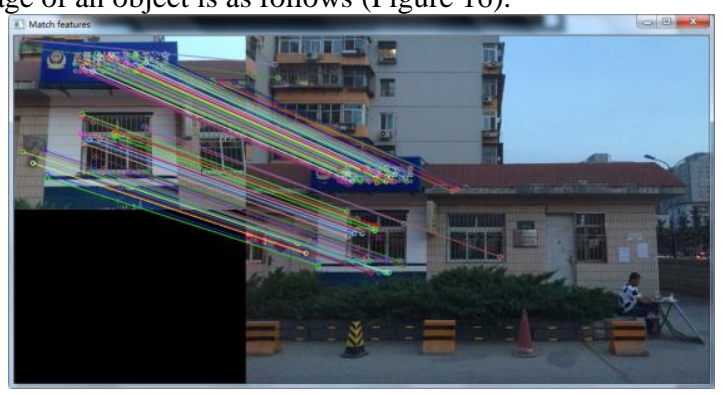

Figure 16. The matching result

Multi-image visualization query result by using the SIFT algorithm is shown. The image of the target area and the image of the search area can be used to visually observe which image is currently selected in the target area. The tree pattern structure in the middle can facilitate the user to query the name attribute, and the matching result on the right side will highlight the location of the target area image in the search area (Figure 17).

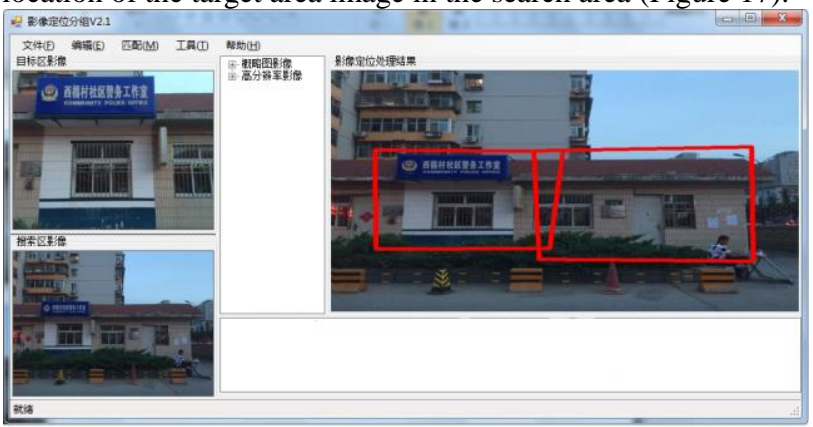

Figure 17. The result of Multi-image visual query

\section{CONCLUSION}

In view of the cultural objects, there are some problems, for example, manually grouping of large-scale image data, manually establishing the relationship between image and point cloud, and query. In order to solve the above problems, we developed a mobile phone APP cooperative photographing system based on Android system. According to its recorded electronic information, large-scale images are automatically grouped. According to image matching technology, the largescale image is divided, and the localization and topological relationship between the local image and the global image are established. For the above positioning results, the matching between the local image and the local point cloud data is established based on the point cloud reflection intensity image corresponding to the global image. For the above topology relationship, Large-scale image visualization query can be achieved. It is proved by experiments that the method of this article is correct and feasible. The study improves the efficiency of image management and automatically establishes a one-toone correspondence between large-scale images and point clouds. It is of great help to provide efficient data for the subsequent Registration of point clouds and images and other problems.

\section{REFERENCES}

Arzt, S., Rasthofer, S., Fritz, C., Bodden, E., Bartel, A., \& Klein, J., et al., 2014. FlowDroid: precise context, flow, field, objectsensitive and lifecycle-aware taint analysis for Android apps. ACM Sigplan Conference on Programming Language Design and Implementation. 49, pp. 259-269.

Barrow, H. G., Tenenbaum, J. M., Bolles, R. C., \& Wolf, H. C., 1977. Parametric correspondence and chamfer matching: two new techniques for image matching. International Joint Conference on Artificial Intelligence, 2, pp.659-663.

Beyer, S., \& Pinzger, M. (2014). A Manual Categorization of Android App Development Issues on Stack Overflow. IEEE International Conference on Software Maintenance and Evolution, pp.531-535.

Brodu, N., \& Lague, D., 2012. 3d terrestrial lidar data classification of complex natural scenes using a multi-scale dimensionality criterion: applications in geomorphology. Isprs Journal of Photogrammetry \& Remote Sensing, 68(1), pp. 121134.

Chin, E., Felt, A. P., Greenwood, K., \& Wagner, D, 2011. Analyzing inter-application communication in android. Plant \& Soil, 269(1-2), pp. 309-320.

Collins, R. T., 1996. Space-sweep approach to true multi-image matching. Cvpr.

Igoe, D. P., Parisi, A., \& Carter, B., 2014. Smartphone-based android app for determining uva aerosol optical depth and direct solar irradiances.Photochemistry \& Photobiology, 90(1), pp. 233-237.

Lim, E. H., \& Suter, D., 2009. 3d terrestrial lidar classifications with super-voxels and multi-scale conditional random fields. Computer-Aided Design, 41(10), pp. 701-710.

Thirion, J. P., 1998. Image matching as a diffusion process: an analogy with maxwell's demons. Medical Image Analysis, 2(3), pp. 243. 